\section{BRAZIULIAN JOURNAL}

OF MEDICAL AND BIOLOGICAL RESHARCH

www.bjournal.com.br
ISSN 0100-879X

Volume 43 (11) 1010-1134 November 2010

BIOMEDICAL SCIENCES

AND

CLINICAL INVESTIGATION

Braz J Med Biol Res, November 2010, Volume 43(11) 1047-1053

doi: 10.1590/S0100-879X2010007500111

Lack of tolerance for the anti-dyskinetic effects of 7-nitroindazole, a neuronal nitric oxide synthase inhibitor, in rats

N. Novaretti, F.E. Padovan-Neto, V. Tumas, C.A. da-Silva and E.A. Del Bel

The Brazilian Journal of Medical and Biological Research is partially financed by
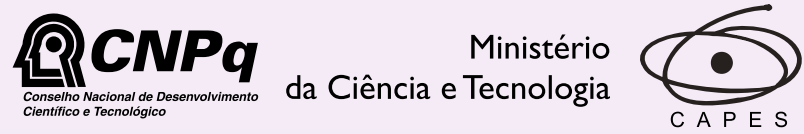

Ministério da Educação

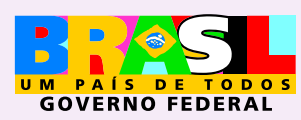

DFAPESP

Institutional Sponsors
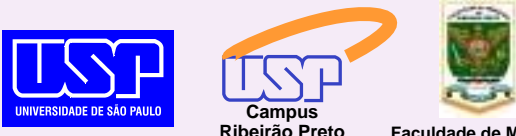$$
\frac{\partial}{\mathrm{an}}
$$

Hots ite of proteomics metabolomics developped by: 


\title{
Lack of tolerance for the anti-dyskinetic effects of 7-nitroindazole, a neuronal nitric oxide synthase inhibitor, in rats
}

\author{
N. Novaretti ${ }^{1,2 *}$, F.E. Padovan-Neto ${ }^{1,2 *}$, V. Tumas ${ }^{1}$, C.A. da-Silva ${ }^{2}$ and E.A. Del Bel ${ }^{1,2}$ \\ ${ }^{1}$ Departamento de Neurociências e Ciências do Comportamento, \\ Faculdade de Medicina de Ribeirão Preto, Universidade de São Paulo, Ribeirão Preto, SP, Brasil \\ 2Departamento de Morfologia, Estomatologia e Fisiologia, \\ Faculdade de Odontologia de Ribeirão Preto, Universidade de São Paulo, Ribeirão Preto, SP, Brasil
}

\begin{abstract}
7-Nitroindazole (7-NI) inhibits neuronal nitric oxide synthase in vivo and reduces I-DOPA-induced dyskinesias in a rat model of parkinsonism. The aim of the present study was to determine if the anti-dyskinetic effect of 7-NI was subject to tolerance after repeated treatment and if this drug could interfere with the priming effect of I-DOPA. Adult male Wistar rats (200-250 g) with unilateral depletion of dopamine in the substantia nigra compacta were treated with I-DOPA (30 mg/kg) for 34 days. On the $1 \mathrm{st}$ day, 6 rats received ip saline and 6 received ip 7-NI $(30 \mathrm{mg} / \mathrm{kg})$ before I-DOPA. From the 2nd to the 26th day, all rats received I-DOPA daily and, from the 27 th to the 34 th day, they also received 7-NI before I-DOPA. Animals were evaluated before the drug and $1 \mathrm{~h}$ after I-DOPA using an abnormal involuntary movement scale and a stepping test. All rats had a similar initial motor deficit. 7-NI decreased abnormal involuntary movement induced by I-DOPA and the effect was maintained during the experiment [before 7-NI, median (interquartile interval), day 26: 16.75 (15.88-17.00); day 28: 0.00 (0.00-9.63); day 29: 13.75 (2.25-15.50); day 30: 0.5 (0.00-6.25); day 31: 4.00 (0.00-7.13), and day 34: 0.5 (0.00-14.63), Friedman followed by Wilcoxon test, vs day 26 , $\mathrm{P}<0.05]$. The response to I-DOPA alone was not modified by the use of 7-NI before the first administration of the drug (I-DOPA vs time interaction, $\mathrm{F} 1,10=1.5, \mathrm{NS}$ ). The data suggest that tolerance to the anti-dyskinetic effects of a neuronal nitric oxide synthase inhibitor does not develop over a short-term period of repeated administration. These observations open a possible new therapeutic approach to motor complications of chronic I-DOPA therapy in patients with Parkinson's disease.
\end{abstract}

Key words: Nitric oxide; Nitric oxide synthase; Parkinson's disease; L-DOPA; Dyskinesia; DOPA priming; 7-Nitroindazole

\section{Introduction}

Nitric oxide (NO) is a gas synthesized by the NO synthase (NOS) enzyme that diffuses locally in the central nervous system to promote vasodilatation and the modulation of neuronal cells, acting as an "atypical neurotransmitter" (1-3). There are three types of NOS: neuronal (nNOS) and endothelial and inducible NOS (4). The principal form expressed in the brain is nNOS and its expression is restricted to neurons.

It has been proposed that NO modulates motor behavior (5-7). NOS inhibitors inhibit hyperlocomotion induced by dopamine agonists in mice and rats $(1,8,9)$. NOS inhibitors also induce catalepsy after systemic or intrastriatal injection, an effect similar to those observed after application of D2 receptor antagonists $(5,10)$. Tolerance has been reported to occur within 4-5 days of continuous treatment with NOS inhibitors in rodents $(5,10,11)$. The phenomenon of tolerance is characterized by impaired responsiveness to the NOS inhibitors, which is, in turn, demonstrated by reduced cataleptic effect that appears after continuous use. NOS inhibitors-induced tolerance to other drugs that induce catalepsy (e.g., haloperidol) is termed cross-tolerance $(5,10,11)$. Although the mechanisms underlying the phenomenon of NOS inhibitor development of tolerance are still poorly defined (5), it may be an important obstacle for the eventual clinical use of these compounds in motor-related disorders particularly if it concerns therapeutic effects rather than side effects.

Dopaminergic denervation may trigger aberrant neuro-

Correspondence: E.A. Del Bel, Departamento de Morfologia, Estomatologia e Fisiologia, Faculdade de Odontologia de Ribeirão Preto, Av. Café, s/n, 14040-904 Ribeirão Preto, SP, Brasil. E-mail: eadelbel@forp.usp.br

*These authors contributed equally to this study.

Received March 12, 2010. Accepted October 1, 2010. Available online October 15, 2010. Published November 12, 2010. 
plastic changes in the striatum, which could interfere with the processing of movement-related information (12-22). L-DOPA is the most effective symptomatic treatment for Parkinson's disease, but its chronic administration leads to the development of motor complications expressed as fluctuations in clinical response and appearance of abnormal involuntary movements also called I-DOPA-induced dyskinesias (12). The mechanisms underlying this phenomenon are unclear (13). Also, it has been suggested that the very first molecular events thought to be responsible for the establishment of dyskinesia are generally grouped under the term of "priming". Priming is classically defined as the process by which the brain becomes sensitized such that administration of a dopaminergic therapy modifies the response to subsequent dopaminergic treatments (22). The emergence of abnormal motor responses to repetitive exposure to dopaminergic drugs only occurs after dopamine depletion (13). The authors suggest that dyskinesia and priming are members of the same continuum, dyskinesia developing with chronicity of the treatment (22).

In recent years, non-dopaminergic symptomatic therapies have been extensively sought for ameliorating I-DOPAinduced dyskinesias (14-16). We have recently shown that 7-nitroindazole (7-NI), a preferential nNOS inhibitor, is able to decrease abnormal involuntary movements (AIMs) in I-DOPA-treated rats subjected to unilateral 6-hydroxydopamine (6-OHDA) lesions of the striatal dopaminergic innervation (17). This model has been extensively used to investigate I-DOPA-induced dyskinesias (18-20). It is still unknown, however, if, similar to its cataleptic effects, repeated treatment with $7-\mathrm{NI}$ would also induce tolerance to the anti-dyskinetic effect after repeated administration $(5,11,21)$. Therefore, the main objective of the present study was to determine if the repeated (8 days) administration of 7-NI would interfere with its anti-dyskinetic effects in an I-DOPA-induced dyskinesia model. Another objective of the present study was to determine if pre-treatment with 7-NI injection before the first I-DOPA administration would interfere with the subsequent I-DOPA-induced dyskinesias, which may have important implications for the therapeutic use of this agent.

\section{Material and Methods}

\begin{abstract}
Animals
Adult male Wistar rats $(200-250 \mathrm{~g})$ were housed in groups of 2 or 3 per cage in a temperature-controlled room $\left( \pm 25^{\circ} \mathrm{C}\right)$, under a 12-h light-dark cycle, with free access to food and water. All experiments were conducted according to the principles and procedures described by the Guidelines for the Care and Use of Mammals in Neuroscience and Behavioral Research (ILAR, USA) and to the Guidelines of the School of Medicine of Ribeirão Preto (University of São Paulo, Brazil). This research project, the institution's animal housing conditions and experimental procedures
\end{abstract}

were approved by the local Animal Ethics Committee (protocol No. 101/2009). All efforts were made to minimize animal number. The behavioral test was always performed from 4:00 to $6: 00 \mathrm{pm}$.

\section{6-Hydroxydopamine lesion}

Stereotaxic surgery was performed as described (23). Rats were anesthetized with tribromoethanol $(0.25 \mathrm{mg} / \mathrm{kg}$ ip, Sigma-Aldrich, USA) and received a single injection of 6-OHDA (Sigma-Aldrich, $16 \mu \mathrm{g}$ in $3 \mu \mathrm{L}$ saline containing $0.05 \%$ ascorbic acid) into the right medial forebrain bundle at the following coordinates (24) relative to bregma: anteroposterior: $-4.4 \mathrm{~mm}$; lateral: $+1.2 \mathrm{~mm}$; dorsoventral: -8.2 $\mathrm{mm}$. The toxin was infused at the rate of $1 \mu \mathrm{L} / \mathrm{min}$, and the cannula was left in place for 3 min before withdrawal. To limit the damage to noradrenergic neurons, desipramine hydrochloride (25 mg/kg ip, Sigma-Aldrich) and pargyline (40 mg/kg ip, Sigma-Aldrich) were administered $30 \mathrm{~min}$ before 6-OHDA injection. Rats were placed in clean cages on warming pads to recover from surgery, after which they were returned to group housing.

\section{Drugs}

L-DOPA(Prolopa dispersive, Hoffman-LaRoche, Brazil) plus benserazide- $\mathrm{HCl}\left(7.5 \mathrm{mg} \cdot \mathrm{kg}^{-1} \cdot \mathrm{day}^{-1}\right)$ were dissolved in physiological saline and given orally by gavage at the dose of $30 \mathrm{mg} \cdot \mathrm{kg}^{-1} \cdot \mathrm{day}^{-1}$. 7-NI (Sigma-Aldrich) was dissolved in $50 \%$ DMSO-saline solution and administered ip at the dose of $30 \mathrm{mg} / \mathrm{kg} 30 \mathrm{~min}$ prior to I-DOPA intake. The doses were based on a previous study using a similar protocol (17). All solutions were prepared immediately prior to use and given in a volume of $2 \mathrm{~mL} / \mathrm{kg}$ body weight.

\section{Behavioral tests}

Abnormal involuntary movements. A trained observer blind to treatment assessed each rat for the presence of the following AIMs (25): axial: lateral flexion and axial rotation of the neck and trunk towards the side contralateral to the lesion; limb: repetitive, rhythmic jerky movements or dystonic posturing of the forelimb on the side contralateral to the lesion; orolingual: tongue protrusion without the presence of food or other objects; locomotor: locomotion to the contralateral side of lesion $(18,20)$. The scores were: 0 , absent; 1 , present for less than half of the observation time; 2, present for more than half of the observation time; 3 , continuous but interrupted by strong sensory stimuli, or 4 , continuous and not interrupted by strong sensory stimuli. Different amplitude scores (ranging from 0, minimal displacement, to 4, maximal displacement) were assigned to forelimb and axial movements. The amplitude of axial AIMs was rated according to the lateral deviation (or torsion) of an animal's neck and trunk from the longitudinal axis of its body, while the amplitude of limb AIMs was rated based on both the magnitude of paw/limb translocation and on the visible involvement of distal versus proximal muscle groups 
(25). Borderline scores $(0.5,1.5,2.5$, and 3.5) were allowed in order to increase the sensitivity of the rating. AlMs were assessed during the pre-test (pre-drug) period and $1 \mathrm{~h}$ after I-DOPA administration, for $1 \mathrm{~min}$, and this was always the first test to be done. The final score was calculated as the sum of all individual movements analyzed (theoretical maximum score $=24$ per evaluation).

Forward stepping test. This is a modified version of the stepping test originally proposed by Olsson et al. (26). Rats were held by the rear part of their torso with one hand with their hind limbs lifted and one forepaw held steady along its trunk by the hand of the experimenter. Then, the animal was moved forward across a table for a distance of $90 \mathrm{~cm}$ within $12 \mathrm{~s}$ (27). During this interval, the numbers of adjusting steps of the weight-bearing forepaw compensating for the straight ahead movement of the body were counted. Each forepaw was tested three times and for each animal the test was begun randomly with the right or left forepaw. The average number of adjusting steps performed in the three trials for each forepaw was used for analysis of motor performance. The performance of each animal was defined according to the relative asymmetry in the motor performance of the forepaws. For this purpose, we calculated a left/right (L/R) index of the number of adjusting steps for each animal. An $\mathrm{L} / \mathrm{R}$ index below 1.0 indicates lower performance with the left forepaw. The animal performance is expressed as the average $L / R$ index obtained in the pre-test (pre-drug) and $1 \mathrm{~h}$ after I-DOPA intake (post-drug) and this was always the second test to be done.

\section{Tissue processing}

Twenty-four hours after the last test, rats were deeply anesthetized with urethane ( $25 \mathrm{mg} / \mathrm{kg}$, Sigma-Aldrich) and then rapidly perfused transcardially with $250 \mathrm{~mL}$ cold saline and $400 \mathrm{~mL} \mathrm{4 \%}$ paraformaldehyde (Sigma-Aldrich) in $0.1 \mathrm{M}$ phosphate buffer, $\mathrm{pH}$ 7.4. Brains were immediately removed, fixed in $4 \%$ paraformaldehyde for $24 \mathrm{~h}$, cryoprotected in $30 \%$ sucrose solution, snap frozen in isopentane $\left(-40^{\circ} \mathrm{C}\right.$, Sigma), and stored at $-70^{\circ} \mathrm{C}$ until use. The tissues were cut into $40-$ $\mu \mathrm{m}$ sections with a freezing microtome. Sections through the striatum and substantia nigra compacta (SNc) were collected in $10 \mathrm{mM}$ phosphate-buffered saline containing
$0.02 \%$ sodium azide and stored at $4^{\circ} \mathrm{C}$ until use.

\section{Immunohistochemistry}

Immunohistochemistry was performed using a standard peroxidase-based method (28). Randomly sampled sections were incubated with a tyrosine hydroxylase $(\mathrm{TH})$ antibody $\left(1: 2000\right.$, Pel Freez, USA) overnight at $4^{\circ} \mathrm{C}$ followed by a biotinylated secondary antibody (Vectastain $A B C$ kit, Vector Laboratories, USA) and HRP-conjugated streptavidin (Vectastain ABC kit, Vector Laboratories). The sections were developed using diaminobenzidine (Sigma-Aldrich) as the chromogen. The slices were mounted on slides and coverslipped for microscopic observation. Structure localization was determined with the help of the Paxinos and Watson atlas (24). Images containing TH immunostaining were digitally captured with an Olympus DP70 camera connected to the microscope.

\section{Experimental design}

Twenty-one days after the stereotaxic surgery all rats were subjected to the stepping test $(N=20)$. Only 6-OHDAlesioned rats presenting an $\mathrm{L} / \mathrm{R}$ index lower than $0.60(\mathrm{~N}=$ 12) were included in the study.

Rats were divided into two well-matched groups ( $N=6$ / group) according to $L / R$ index and received either vehicle ( $2 \mathrm{~mL} / \mathrm{kg}$, ip; vehicle/l-DOPA group) or $7-\mathrm{NI}$ (30 mg/kg, ip; 7-NI/I-DOPA group) followed 30 min later by I-DOPA (30 $\mathrm{mg} / \mathrm{kg}+7.5 \mathrm{mg} / \mathrm{kg}$ of benserazide, by gavage). From the second day on, all animals started receiving single daily administrations of I-DOPA for an additional 24 days. From day 27 to 34, they received an ip injection of 7-NI $30 \mathrm{~min}$ before the daily I-DOPA intake (30 mg/kg; Figure 1). Measurements of AIM scores were performed at days 1, 5, 13, $26,28,29,30,31$, and 34 of treatment. The stepping test was performed at days $1,5,13,26,29$, and 34 of treatment (Figure 1).

\section{Statistical analysis}

Data are reported as means \pm SEM. Data from the stepping test were analyzed by repeated measure multifactorial analysis of variance, with the factors being groups (vehicle and 7-NI), time and I-DOPA (before and after). Specific

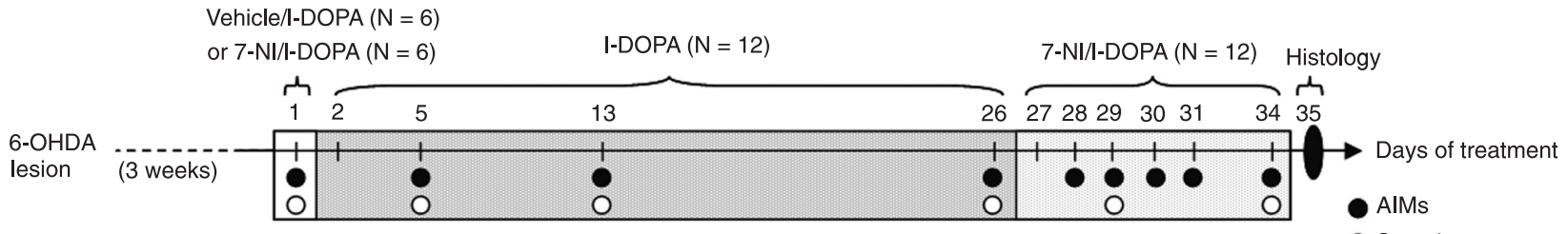

Figure 1. Diagram summarizing the order of the treatments and behavioral tests. Tests started 3 weeks after 6-OHDA lesion. Abnormal involuntary movements (AIMs) and stepping tests were assessed on day 1 after acute administration of vehicle/l-DOPA or 7-NI/I-DOPA. AIMs were assessed at chronic times during I-DOPA (from days 2 to 26) or 7-NI/I-DOPA (from days 27 to 34) administration. Histological procedures were performed on day 35 of treatment. 6-OHDA $=6$-hydroxydopamine; 7- $\mathrm{NI}=7$-nitroindazole. 
MANOVA was also performed to determine the effects of vehicle versus 7-NI treatment on the first day and to compare the effects of I-DOPA on the last administration alone (day 26) versus the subsequent administration under the influence of 7-NI (from day 27 on). In case of significant interactions, post hoc comparisons were made using the Sidak test.

The effectiveness of I-DOPA in inducing AIMs at each assessment point was verified by the non-parametric Wilcoxon test. AIMs induced by I-DOPA were analyzed by the Mann-Whitney (first day) or Friedman test followed, when significant, by the Wilcoxon test for pairwise comparisons. The level of significance was set at $P<0.05$ in all analyses.

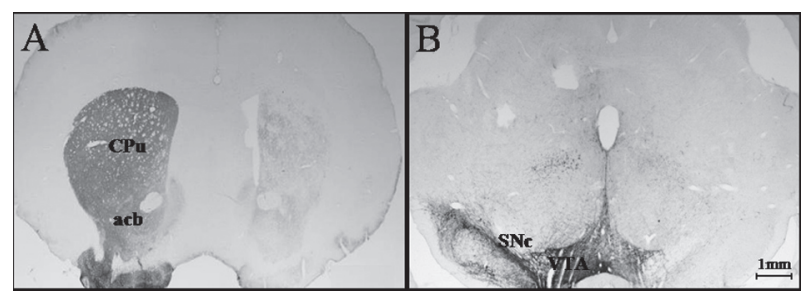

Figure 2. Photomicrograph of a cross-section through the striatum (A) and mesencephalon (B) of a representative unilateral 6-hydroxydopamine-lesioned rat with absence of tyrosine hydroxylase immunoreactive fibers/cells. $\mathrm{CPu}=$ caudate-putamen; $\mathrm{acb}=$ accumbens nuclei; $\mathrm{VTA}=$ ventral tegmental area; $\mathrm{SNc}=$ substantia nigra compacta.

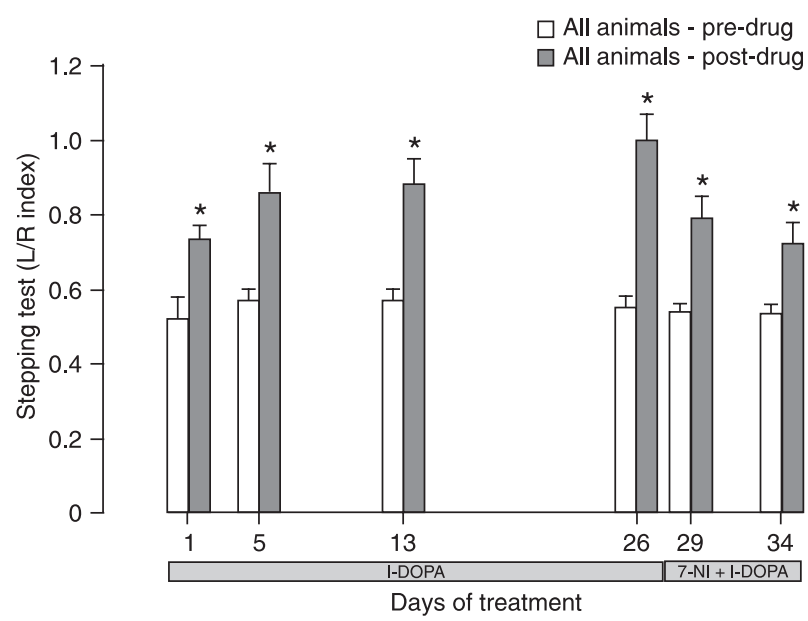

Figure 3. Effects of I-DOPA (30 mg/kg) administration on stepping test performance of 6-OHDA-lesioned rats $(\mathrm{N}=12)$. The stepping test performance is reported as means (+ SEM) left/ right paw $(L / R)$ index obtained before and $1 \mathrm{~h}$ after I-DOPA intake. From day 1 to day 26 the animals received only I-DOPA. Starting on day 27 until day 34 they received an ip injection of 7-NI (30 mg/kg) $30 \mathrm{~min}$ before I-DOPA administration. 6-OHDA = 6-hydroxydopamine; 7 - $\mathrm{NI}=7$-nitroindazole. ${ }^{*} \mathrm{P}<0.05$ vs predrug (MANOVA)

\section{Results}

Rats with 6-OHDA lesions ( $N=12)$ showed a complete absence of immunochemical reaction $\mathrm{TH}$ in the right striatum and in the SNc ipsilateral to the lesion (Figure 2).

On the first day, I-DOPA caused a significant improvement in the forward stepping test $(F 1,10=7.6, P=0.02)$ and this effect was not modified by 7-NI (treatment factor, F1,10 $=0.27$, treatment versus I-DOPA, F1, $10=0.67$, NS; Figure $3)$. No difference between the groups treated with 7-NI or vehicle on day 1 was found in subsequent measurements (treatment factor, $\mathrm{F} 1,10=0.38 \mathrm{NS}$ ). As a consequence, the results were shown as a single group.

The effect of I-DOPA was maintained throughout the experiment $(F 1,10=53.06, P<0.001)$ and did not change along time (time factor, F5,6 = 2.75, P = 0.12, NS; I-DOPA versus time, F5, $6=0.98$, NS; Figure 3). Comparison of the effects of I-DOPA on day 26 (before 7-NI) and days 29 and 34 (after 7-NI) showed that 7-NI treatment did not modify the effects of I-DOPA(I-DOPA versus time interaction, F1,10 $=1.5$, NS; Figure 3).

L-DOPA induced AIMs in all measurements (Wilcoxon test, $P<0.05)$ except those made on day $28(P=0.068$; Figure 4). 7-NI decreased AIMs induced by I-DOPA on day 1 (Mann-Whitney test, $P=0.005$; Figure 5). Similar to the stepping test, no difference between groups treated with 7-NI or vehicle on day 1 was found in subsequent measurements $(P>0.05)$ and the results are shown as a single group. I-DOPA effects increased from day 1 to days

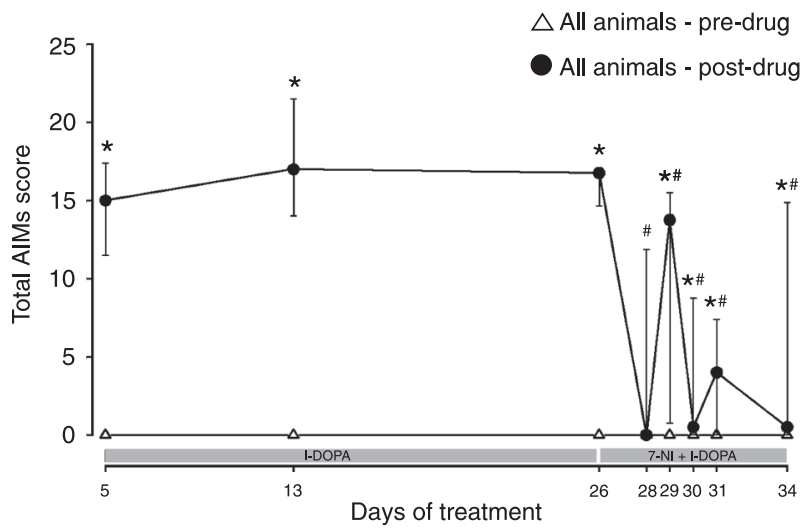

Figure 4. Effects of I-DOPA ( $30 \mathrm{mg} / \mathrm{kg}$ ) administration on total abnormal involuntary movements (AIMs) of 6-OHDA-lesioned rats $(\mathrm{N}=12)$. AlMs were scored $60 \mathrm{~min}$ before or after I-DOPA intake at days 5, 13, 26, 28, 29, 30, 31, and 34 of treatment. From day 1 to day 26 the animals received only I-DOPA. Starting on day 27 until day 34 they received an ip injection of 7-NI $(30 \mathrm{mg} /$ $\mathrm{kg}) 30 \mathrm{~min}$ before I-DOPA administration. Data are reported as median \pm interquartile interval. 6-OHDA = 6-hydroxydopamine; 7-NI $=7$-nitroindazole. ${ }^{*} \mathrm{P}<0.05$ vs pre-drug (Wilcoxon signed rank test), ${ }^{\#} \mathrm{P}<0.05$ vs day 26 (Friedman followed by Wilcoxon test). 
5,13 , and 26 (Friedman test followed by the Wilcoxon test; $\mathrm{P}<0.05)$. 7-NI induced a significant decrease in I-DOPAinduced AIMs that was maintained until the last measurement (Friedman test followed by the Wilcoxon test, versus day $26 ; \mathrm{P}<0.05)$.

\section{Discussion}

The present results showed that repeated treatment with 7-NI was able to decrease I-DOPA-induced dyskinesia in a model of Parkinson's disease. The above mentioned disorder requires chronic treatment and thus repeated drug applications.

Unilateral lesions induced by 6-OHDA injections into the medial forebrain bundle have been extensively used to investigate the neurobiology of Parkinson's disease $(18,20)$. As indicated by $\mathrm{TH}$ immunohistochemistry, in the present study this experimental approach was able to induce severe striatal dopamine denervation.

The motor impairments caused by these lesions can be measured by the forward stepping test and are reflected as an asymmetry between adjusting steps from the lesioned and non-lesioned sides (27). Accordingly, unpublished results (Padovan-Neto FE, Del Bel EA) from our laboratory have shown that an $L / R$ index lower than 0.60 indicates a $93.4 \%$ probability of reflecting a severe right striatal dopaminergic depletion (>95\%). This result was confirmed by TH immunohistochemistry. As shown in Figure 3, I-DOPA was able to improve motor performance, increasing the $L / R$ index throughout the experiment.

Our experiment reproduced a previously reported model (17) of I-DOPA-induced dyskinesias in hemiparkinsonian rats that used a daily dosage of I-DOPA about three times higher than that usually taken by patients with Parkinson's disease, and the drug is given to animals all at once by gavage. Because of this, our animals presented significant AIMs at the first I-DOPA intake (17). These motor complications are clinically relevant and the mechanisms underlying them are still not completely understood (29). It is argued that a significant lack of presynaptic dopaminergic terminals in the striatum would enhance the variability of striatal dopamine levels due to loss of capacity to locally uptake I-DOPA and store dopamine (30). Probably, the main sites of decarboxylation of exogenous I-DOPA to dopamine, when nigrostriatal dopaminergic neurons are damaged, are the serotonergic striatal terminals $(29,31)$. This alternative pathway is probably poorly regulated and may result in an aberrant handling of I-DOPA in the striatum.

There is also evidence that non-physiologic pulsatile striatal dopaminergic stimulation induces activation of nuclear signaling pathways that leads to the development of postsynaptic plastic changes in basal ganglia circuits that could cause an imbalance of cortical-striatal loops and facilitate abnormal involuntary movements (29). Indirect striatopallidal projections may also be abnormally active,

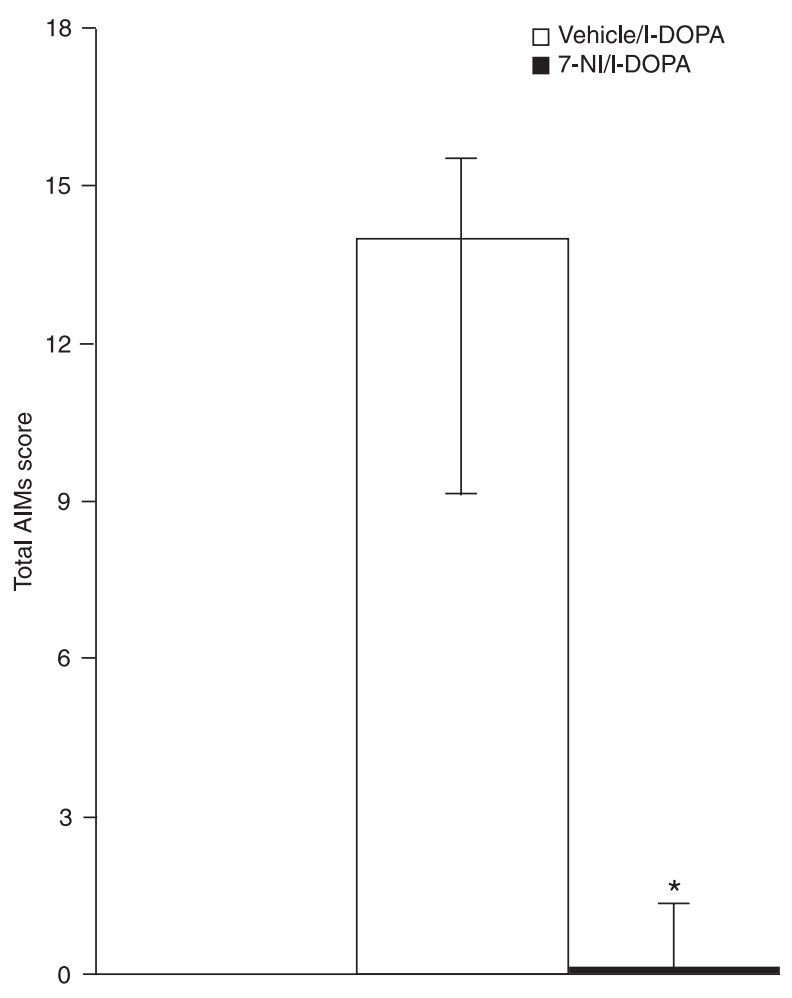

Figure 5. Effects of I-DOPA (30 mg/kg) administration on total abnormal involuntary movements (AIMs) of 6-OHDA-lesioned rats $(\mathrm{N}=12)$ on the first day of treatment. AIMs were scored 60 min before or after I-DOPA intake. Thirty minutes before I-DOPA administration the animals ( $N=6 /$ group) received ip injections of vehicle or $7-\mathrm{NI}(30 \mathrm{mg} / \mathrm{kg})$. Data are reported as median \pm interquartile interval. 6-OHDA = 6-hydroxydopamine; 7- $\mathrm{NI}=7$-nitroindazole. ${ }^{*} P<0.05$ (Mann-Whitney test).

probably due to excessive glutamatergic excitation after striatal dopaminergic denervation (32). Drugs that block either N-methyl-D-aspartate (NMDA) or alpha-amino-3hydroxy-5-methyl-4-isoxazole propionic acid (AMPA) receptors tend to ameliorate dyskinesias in Parkinson models and patients (29).

Nitrergic mechanisms may also contribute to the pathogenesis of I-DOPA-induced dyskinesias. Animal experiments have suggested that striatal dopaminergic lesions and chronic treatment with I-DOPA increase the synthesis of $\mathrm{NO}$ in basal ganglia (23,33-35), while the cerebrospinal fluid concentration of nitrate and nitrite is elevated in patients with Parkinson's disease receiving dopamine replacement (36). The NO second messenger cyclic guanosine monophosphate (cGMP) is increased in the serum of Parkinson's disease patients (37) and is regulated by I-DOPA itself (33). Chronic I-DOPA treatment causes overexpression of FosB/ $\triangle \mathrm{FosB}$ in neurons of the striatal direct output pathway and in NOS interneurons in lesioned areas of the striatum $(35,38)$. Confirming a previ- 
ous report (17), 7-NI inhibited I-DOPA-induced dyskinesias. $7-\mathrm{NI}$ is a preferential neuronal NOS inhibitor that has been shown to attenuate NO striatal efflux evoked by electrical stimulation of the substantia nigra compacta after systemic administration (39).

NO has been proposed to modulate striatum function, changing its input-output relationship and producing a functionally significant impact on its target neurons (40). Striatal NO-producing interneurons are regulated by phasic dopamine transmission and NO released from striatal interneurons facilitates the concurrent release of dopamine (40). Probably reflecting the influence of NO on dopamine function, previous studies have shown that systemic or intrastriatal administration of NOS inhibitors causes catalepsy in rodents that is additive to that induced by haloperidol, a dopamine-D2 receptor antagonist (5). This cataleptic effect of NOS inhibition, however, suffers tolerance after 4 days of repeated administration $(5,11,21)$. Although the mechanisms of this tolerance is still unknown, recent evidence suggests that it does not depend on changes in D2 receptors but rather on plastic changes in nNOS neurons resulting in partial recovery of $\mathrm{NO}$ formation in the striatum (21). The present results show that this rapid tolerance does not develop to the anti-dyskinetic effects of this drug, at least during the treatment period ( 8 days). Moreover, the fact that the positive motor effects of I-DOPA in the forward stepping test were preserved during 7-NI treatment suggests that any direct interference of NOS inhibition with striatal levels of dopamine was able to prevent AIMs without significantly changing normal motor function.

Although the concept of I-DOPA-induced "priming" has been recently criticized (22), it has been suggested that, in 6-OHDA-lesioned rats, a first "priming" dose of a dopamine agonist sensitizes the animal to the effect of a

\section{References}

1. Hara MR, Snyder SH. Cell signaling and neuronal death. Annu Rev Pharmacol Toxicol 2007; 47: 117-141.

2. Vincent $\mathrm{SR}$, Kimura $\mathrm{H}$. Histochemical mapping of nitric oxide synthase in the rat brain. Neuroscience 1992; 46: 755-784.

3. Kiss JP, Vizi ES. Nitric oxide: a novel link between synaptic and nonsynaptic transmission. Trends Neurosci 2001; 24: 211-215.

4. Garthwaite J. Concepts of neural nitric oxide-mediated transmission. Eur J Neurosci 2008; 27: 2783-2802.

5. Del Bel EA, Guimaraes FS, Bermudez-Echeverry M, Gomes MZ, Schiaveto-de-souza A, Padovan-Neto FE, et al. Role of nitric oxide on motor behavior. Cell Mol Neurobiol 2005; 25: 371-392.

6. Salum C, Raisman-Vozari R, Michel PP, Gomes MZ, Mitkovski M, Ferrario JE, et al. Modulation of dopamine uptake by nitric oxide in cultured mesencephalic neurons. Brain Res 2008; 1198: 27-33.

7. West AR, Galloway MP, Grace AA. Regulation of striatal dopamine neurotransmission by nitric oxide: effector pathways subsequent challenge with dopamine agonists. Moreover, dyskinesia and priming have been proposed to share similar mechanisms, with dyskinesia developing with chronic treatment (22). We evaluated how "priming", a phenomenon displaying neurochemical and behavioral features peculiar to a sensitized abnormal motor response in dopaminedenervated rats, depends on the pre-treatment with 7-NI injection before the first I-DOPA administration. Because we wanted to study this effect, we did not use the rotational behavior induced by amphetamine or apomorphine as an indicator for striatal dopamine depletion. Nevertheless, in the present study, treatment with $7-\mathrm{NI}(30 \mathrm{mg} / \mathrm{kg})$ before the first dose of I-DOPA did not affect priming magnitude. This result indicates that NOS inhibition does not seem to play a fundamental role in determining the outcome of subsequent motor responses elicited by dopamine receptor stimulation.

The present results suggest that the anti-dyskinetic effects of NOS inhibition at the dose tested do not significantly impair normal motor performance and do not suffer tolerance over a period of 8 days. These observations open a new possible therapeutic approach to motor complications of chronic I-DOPA therapy in patients with Parkinson's disease.

\section{Acknowledgments}

We acknowledge the helpful technical support of Daniele Tavares. We gratefully acknowledge the helpful support for statistical analysis and manuscript discussion of Prof. Dr. Francisco S. Guimarães (Pharmacology, FMRP, USP). Research supported by CAPES/COFECUB, FAPESP/ INSERM, FAPESP, and CNPq. N. Novaretti received a PIBIC-CNPq fellowship. and signaling mechanisms. Synapse 2002; 44: 227-245.

8. Dzoljic E, De Vries R, Dzoljic MR. New and potent inhibitors of nitric oxide synthase reduce motor activity in mice. Behav Brain Res 1997; 87: 209-212.

9. Sandi C, Venero C, Guaza C. Decreased spontaneous motor activity and startle response in nitric oxide synthase inhibitor-treated rats. Eur J Pharmacol 1995; 277: 89-97.

10. Marras RA, Martins AP, Del Bel EA, Guimaraes FS. L-NOARG, an inhibitor of nitric oxide synthase, induces catalepsy in mice. Neuroreport 1995; 7: 158-160.

11. Del Bel EA, Guimaraes FS. Sub-chronic inhibition of nitricoxide synthesis modifies haloperidol-induced catalepsy and the number of NADPH-diaphorase neurons in mice. Psychopharmacology 2000; 147: 356-361.

12. Rascol O, Payoux P, Ory F, Ferreira JJ, Brefel-Courbon C, Montastruc JL. Limitations of current Parkinson's disease therapy. Ann Neurol 2003; 53 (Suppl 3): S3-S12.

13. Jenner P. Molecular mechanisms of L-DOPA-induced dyskinesia. Nat Rev Neurosci 2008; 9: 665-677. 
14. Grondin R, Bedard PJ, Hadj TA, Gregoire L, Mori A, Kase $H$. Antiparkinsonian effect of a new selective adenosine A2A receptor antagonist in MPTP-treated monkeys. Neurology 1999; 52: 1673-1677.

15. Papa SM, Auberson YP, Greenamyre JT. Prolongation of levodopa responses by glycineB antagonists in parkinsonian primates. Ann Neurol 2004; 56: 723-727.

16. Papa SM, Chase TN. Levodopa-induced dyskinesias improved by a glutamate antagonist in Parkinsonian monkeys. Ann Neurol 1996; 39: 574-578.

17. Padovan-Neto FE, Echeverry MB, Tumas V, Del-Bel EA. Nitric oxide synthase inhibition attenuates L-DOPA-induced dyskinesias in a rodent model of Parkinson's disease. Neuroscience 2009; 159: 927-935.

18. Cenci MA, Lee CS, Bjorklund A. L-DOPA-induced dyskinesia in the rat is associated with striatal overexpression of prodynorphin- and glutamic acid decarboxylase mRNA. Eur $J$ Neurosci 1998; 10: 2694-2706.

19. Cenci MA, Whishaw IQ, Schallert T. Animal models of neurological deficits: how relevant is the rat? Nat Rev Neurosci 2002; 3: 574-579.

20. Lundblad M, Andersson M, Winkler C, Kirik D, Wierup N, Cenci MA. Pharmacological validation of behavioural measures of akinesia and dyskinesia in a rat model of Parkinson's disease. Eur J Neurosci 2002; 15: 120-132.

21. Del-Bel EA, Guimaraes FS, Joca SR, Echeverry MB, Ferreira FR. Tolerance to the cataleptic effect that follows repeated nitric oxide synthase inhibition may be related to functional enzymatic recovery. J Psychopharmacol 2010; 24: 397-405

22. Nadjar A, Gerfen CR, Bezard E. Priming for I-dopa-induced dyskinesia in Parkinson's disease: a feature inherent to the treatment or the disease? Prog Neurobiol 2009; 87: 1-9.

23. Gomes MZ, Del Bel EA. Effects of electrolytic and 6-hydroxydopamine lesions of rat nigrostriatal pathway on nitric oxide synthase and nicotinamide adenine dinucleotide phosphate diaphorase. Brain Res Bull 2003; 62: 107-115.

24. Paxinos G, Watson W. The rat brain in stereotaxic coordinates. 4th edn. San Diego: Academic Press; 21998.

25. Winkler C, Kirik D, Bjorklund A, Cenci MA. L-DOPA-induced dyskinesia in the intrastriatal 6-hydroxydopamine model of parkinson's disease: relation to motor and cellular parameters of nigrostriatal function. Neurobiol Dis 2002; 10: 165186.

26. Olsson M, Nikkhah G, Bentlage C, Bjorklund A. Forelimb akinesia in the rat Parkinson model: differential effects of dopamine agonists and nigral transplants as assessed by a new stepping test. J Neurosci 1995; 15: 3863-3875.

27. Chang JW, Wachtel SR, Young D, Kang UJ. Biochemical and anatomical characterization of forepaw adjusting steps in rat models of Parkinson's disease: studies on medial forebrain bundle and striatal lesions. Neuroscience 1999; 88: 617628.
28. Gomes $M Z$, Raisman-Vozari R, Del Bel EA. A nitric oxide synthase inhibitor decreases 6-hydroxydopamine effects on tyrosine hydroxylase and neuronal nitric oxide synthase in the rat nigrostriatal pathway. Brain Res 2008; 1203: 160169.

29. Cenci MA, Lindgren HS. Advances in understanding LDOPA-induced dyskinesia. Curr Opin Neurobiol 2007; 17: 665-671.

30. Chase TN, Baronti F, Fabbrini G, Heuser IJ, Juncos JL, Mouradian MM. Rationale for continuous dopaminomimetic therapy of Parkinson's disease. Neurology 1989; 39: 7-10.

31. Lopez A, Munoz A, Guerra MJ, Labandeira-Garcia JL. Mechanisms of the effects of exogenous levodopa on the dopamine-denervated striatum. Neuroscience 2001; 103: 639-651.

32. Chase TN. Striatal plasticity and extrapyramidal motor dysfunction. Parkinsonism Relat Disord 2004; 10: 305-313.

33. Chalimoniuk M, Langfort J. The effect of subchronic, intermittent L-DOPA treatment on neuronal nitric oxide synthase and soluble guanylyl cyclase expression and activity in the striatum and midbrain of normal and MPTP-treated mice. Neurochem Int 2007; 50: 821-833.

34. Chalimoniuk M, Lukacova N, Marsala J, Langfort J. Alterations of the expression and activity of midbrain nitric oxide synthase and soluble guanylyl cyclase in 1-methyl-4-phenyl1,2,3,6-tetrahydropyridine-induced Parkinsonism in mice. Neuroscience 2006; 141: 1033-1046.

35. Pavon N, Martin AB, Mendialdua A, Moratalla R. ERK phosphorylation and FosB expression are associated with L-DOPA-induced dyskinesia in hemiparkinsonian mice. Biol Psychiatry 2006; 59: 64-74.

36. Molina JA, Jimenez-Jimenez FJ, Navarro JA, Vargas C, Gomez P, Benito-Leon J, et al. Cerebrospinal fluid nitrate levels in patients with Parkinson's disease. Acta Neurol Scand 1996; 93: 123-126.

37. Chalimoniuk M, Stepien A. Influence of the therapy with pergolide mesylate plus L-DOPA and with L-DOPA alone on serum cGMP level in PD patients. Pol J Pharmacol 2004; 56: 647-650.

38. Cenci MA, Tranberg A, Andersson M, Hilbertson A. Changes in the regional and compartmental distribution of FosB- and JunB-like immunoreactivity induced in the dopamine-denervated rat striatum by acute or chronic L-dopa treatment. Neuroscience 1999; 94: 515-527.

39. Sammut S, Dec A, Mitchell D, Linardakis J, Ortiguela M, West AR. Phasic dopaminergic transmission increases NO efflux in the rat dorsal striatum via a neuronal NOS and a dopamine $D(1 / 5)$ receptor-dependent mechanism. NeuroOpsychopharmacology 2006; 31: 493-505.

40. West AR, Grace AA. The nitric oxide-guanylyl cyclase signaling pathway modulates membrane activity states and electrophysiological properties of striatal medium spiny neurons recorded in vivo. J Neurosci 2004; 24: 1924-1935. 